\title{
Ultrastructural Pathological Study of Left Ventricular Myocardium in Patients with Isolated Rheumatic Mitral Stenosis with Normal or Abnormal Left Ventricular Function
}

\author{
Ying-Shiung LeE, M.D., F.C.C.P., F.I.C.A. and \\ Ching-Ping LEe, M.D.
}

\section{Summary}

An electron microscopic study of left ventricular myocardium was carried out in 15 patients who had isolated rheumatic mitral stenosis, with particular reference to the relation among ultrastructural pathological findings, the severity of mitral stenosis and left ventricular function. They were divided into 2 groups based on left ventricular performance evaluated by 2-dimensional echocardiography and angiocardiography. The severity of mitral stenosis was determined by hemodynamic data and mitral valve areas measured by 2 -dimensional echocardiography. Regardless of the level of left ventricular contractile function we consistently demonstrated varying degrees of ultrastructural pathological alterations of left ventricular muscle cells, involving the myofibrils, mitochondria, nuclei and other elements of the sarcoplasm and membranes surrounding the myocardial cells in all specimens examined. The ultrastructural pathological findings did not correlate with the severity of mitral stenosis reflected in the echocardiographic and hemodynamic data. However, those patients with abnormal left ventricular function always exhibited more extensive loss of myofibrils resulting from either disproportion of the mitochondria-to-myofibril ratio or myofibrillar degeneration. The present investigation provides the morphological data at the ultrastructural level to support the widely held concept of a myocardial factor i.e., the extent of myocardial involvement by the rheumatic process as the basic pathogenetic mechanism responsible for left ventricular dysfunction in patients with isolated rheumatic mitral stenosis. Furthermore, it is suggestcd that pathological alterations of myocardial ultrastructure were related to the extent of myocardial involvement by the rheumatic process rather than being structural adaptations in response to the hemodynamic derangement.

From the Cardiovascular Division, Department of Internal Medicine, Ghang Gung Memorial Hospital, Taipei, Taiwan, Republic of China.

Mailing address: Ying-Shiung Lee, M.D., Director in Cardiovascular Division, Department of Internal Medicine, Ghang Gung Memorial Hospital, 199 Tun Hwa North Road, Taipei, Taiwan, Republic of China.

Received for publication August 28, 1989.

Accepted January 31, 1990. 


\title{
Additional Indexing Words:
}

Mitral stenosis Myocardial ultrastructure

\begin{abstract}
A
number of reports have stressed the fact that patients with isolated rheumatic mitral stenosis have evidence of impairment in left ventricular function. ${ }^{1{ }^{1-8}}$ Furthermore, left ventricular dysfunction may be a contributing factor in some patients who have an unsatisfactory clinical response to surgical repair of a mitral valve lesion.9) Although abnormal function of the left ventricle is evident in some cases with isolated mitral stenosis, the mechanism of this dysfunction remains unexplained. Some investigators have felt that the impairment of left ventricular contractility is related to the severity of mitral stenosis reflected in left atrial and right ventricular dilatation..$^{6,8)}$ However, histopathologic studies in patients with mitral stenosis have shown that the failure of improvement after mitral commissurotomy was related to the extent of myocardial involvement by the rheumatic process. ${ }^{9}$ The presence of left ventricular dysfunction in isolated mitral stenosis points toward a myocardial factor. At present there is still no systematic report dealing with the alterations of myocardial ultrastructure in rheumatic mitral stenosis. Thus, an objective of the present study was to report electron microscopic observations of pathologic changes of left ventricular myocardium in patients with isolated mitral stenosis. Particular attention was directed to the correlation between ultrastructural pathological findings and the severity of mitral stenosis. It is hoped that the present communication can provide further knowledge regarding the pathogenetic mechanism responsible for abnormal left ventricular function in patients with isolated rheumatic mitral stenosis.
\end{abstract}

\section{Materials and Methods}

Selection of patients: Fifteen consecutive patients with isolated rheumatic mitral stenosis were entered into the study. They were 4 males and 11 females, ranging in age from 25 to 55 years. In all patients the diagnosis established by clinical data including chest roentgenogram, electrocardiography, echocardiography and cardiac catheterization with cineangiocardiography and selective coronary arteriography was further confirmed by operative and histopathological findings. The patients were divided into 2 groups based on global function of the left ventricle evaluated by 2-dimensional echocardiography and left ventricular cineangiocardiography. Group A included 9 patients with adequate left ventricular performance (ejection fraction $>50 \%$ ). Group $B$ consisted of 6 patients with abnormal left ven- 
tricular contractile function (ejection fraction $<45 \%$ ). The normal range of left ventricular ejection fraction in our laboratory is $49-76 \%$. In all patients left atrial and left ventricular dimensions were determined from Mmode echocardiography while mitral valve areas were measured by means of 2-dimensional echocardiography. ${ }^{10}$ ) Hemodynamic data including pulmonary arterial wedge pressure and left ventricular pressure were measured during cardiac catheterization. Coronary arteriography showed normal or nearly normal coronary arteries without significant stenosis in all patients. There was no evidence of myocardial infarction on electrocardiogram. Informed consent was obtained from all patients. Endomyocardial biopsies of the left ventricular myocardium were performed during cardiac catheterization. No complications occurred during or after the biopsies. Three to five samples were obtained at the time of each biopsy. The samples measured 2 to $3 \mathrm{~mm}$ in the greatest dimension. The time from the closure of the jaws of the bioptome on the tissue to the submersion in the fixative solution was less than $10 \mathrm{sec}$.

Preparation for electron microscopy: The specimens obtained from left ventricular myocardium were instantly fixed in 3\% phosphate-buffered glutaraldehyde $(\mathrm{pH}$ 7.4) for 2 hours. The specimens were then rinsed in several changes of cold phosphate buffer and postfixed in $2 \%$ phosphatebuffered osmium tctroxide for an additional 2 hours. After fixation, the tissues were dehydrated in graded concentrations of chilled ethanol and were embedded in Epon-Araldite. The specimens were sectioned with a Sorval MT-2B Porter-Blum ultramicrotome using diamond knives and stained with uranyl acetate and lead citrate. The ultrathin sections were examined with a Hitachi H-500 electron microscope operating at 75 or $100 \mathrm{KV}$.

Statistical analysis: All data were statistically analysed according to the Student's paired t-test. A p value of less than 0.05 was accepted as the limit of significance.

\section{RESUlts}

Pathological alterations of myocardial ultrastructures: It is well known that all the structures forming the muscle cells and the tissues around them, namely the nerves, blood vessels, fibroblasts and collagen, may participate in the pathological process. The present report focused on the pathological changes which took place in the muscle cells involving the myofibrils, mitochondria, nuclei and other elements of the sarcoplasm and the membranes surrounding the cells. Regardless of whether the patients had normal or abnormal left ventricular function, various degrees of pathological alterations of myocardial 


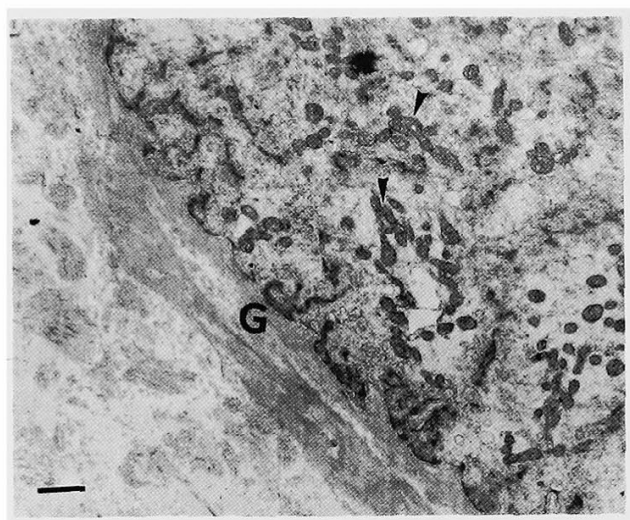

Fig. 1.

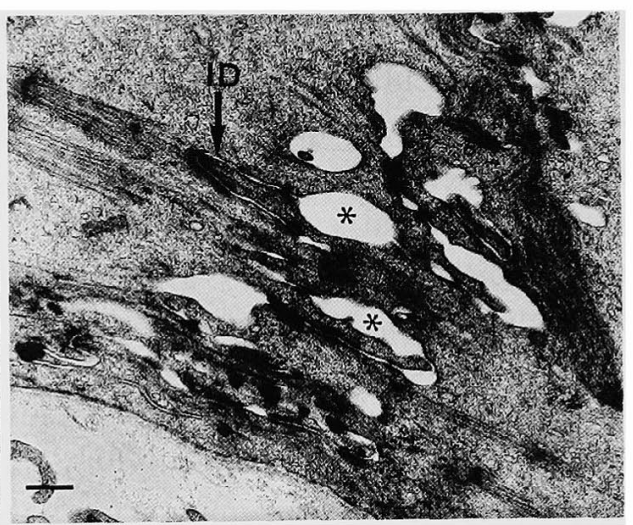

Fig. 2.

Fig. 1. Irregular thickening of the glycocalyx (G) associated with degenerative change of myocardial cell. Random distribution of small mitochondria (arrow heads) with deposition of granular material in the sarcoplasm is clearly demonstrated. Horizontal bar $=1 \mu \mathrm{m}(\times 10,000)$.

Fig. 2. Focal separation of the intercalated discs (asterisks). ID=intercalated disc. Horizontal bar $=0.5 \mu \mathrm{m}(\times 20,000)$.

subcellular structures were consistently observed in all specimens examined. The findings will be described in detail in the following.

1. Sarcolemma-Glycocalyx Complex and Intercalated Discs. The sarcolemma-glycocalyx complex of myocardial cells is normally comprised of three distinct layers i.e., two electron-dense layers (external lamina of the glycocalyx and the sarcolemma) separated by an electron-lucent central zone (surface coat of the glycocalyx) when viewed with an electron microscope. ${ }^{11)}$ In contrast to the normal findings, the sarcolemma-glycocalyx complex of muscle cells observed in the diseased myocardium obtained from patients with chronic rheumatic mitral stenosis often showed irregular thickening of the glycocalyx with apparent loss of a triple-layered structure, particularly in the cells with advanced degenerative changes (Fig. 1). Lamination and/or wide splitting of the glycocalyx were not infrequently seen. Occasionally, a defective sarcolemma which showed breaks and discontinuities was observed. Focal separation of intercalated discs always associated with disruption and detachment of the disc membrane structures was a common finding (Fig. 2). In some instances the intercalated discs showed extensive separation involving not only fascia and macula adherans but also the nexus junction.

2. Myofibrils. Various degrees of degenerative changes of the myofibrils involving the myofilaments and the Z-lines were consistently observed in all specimens examined (Fig. 3). The myofibrils were often reduced in 


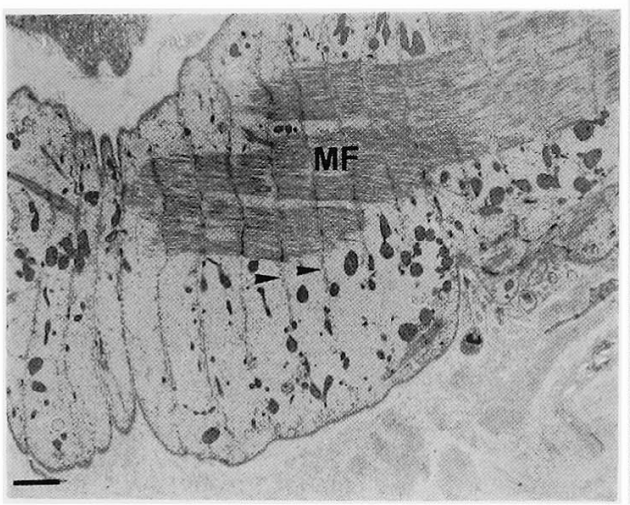

Fig. 3.

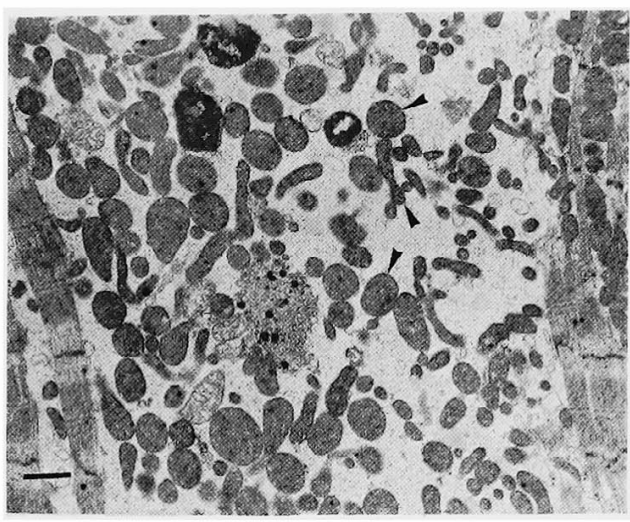

Fig. 4.

Fig. 3. Apparent loss of myofilaments involving several sarcomeres with the remains of $\mathrm{Z}$-lines (arrow heads). $\mathrm{MF}=$ myofibril. Horizontal bar $=2$ $\mu \mathrm{m}(\times 5,000)$.

Fig. 4. An accumulation of the mitochondria (arrow heads) with marked variation in their size and shape. Horizontal bar $=1 \mu \mathrm{m}(\times 10,000)$.

diameter with a relative increase of the intervening sarcoplasm. Loss of myofilaments involved one or more sarcomeres and was sometimes more apparent in the I-band. Part of the Z-line persisted after the filaments of the I-band disappeared. These degenerative changes of the myofibrils may be reflected in the configuration of the muscle cells, the surface of which often became very indented or presented sarcoplasmic projections. Severe degeneration of the myofibrils resulted in the formation of the sarcoplasmic masses which contained many glycogen granules, mitochondria, occasional nuclei and remains of degenerated myofibrils. Eventually, the myofibrils disappeared from a considerable portion of the cells with consequent diminution in their size. Disarray and loss of myofibrils were accompanied by changes in the shape and size of the muscle cells. The Z-lines frequently exhibited structural changes and became irregular in shape and size and often appeared to extend into the I- and A-bands. Thickening and distortion and even fragmentation of the Z-lines were also commonly seen.

3. Mitochondria. The more common mitochondrial changes observed in all specimens were variation in their number, size and shape (Fig. 4). Often their ultrastructures were also altered. The number of mitochondria might be increased or severely reduced. An increase in number occurred in the early stage of disease, while in degenerating cells their number was reduced. Eventually the mitochondria disappeared from the very severely degenerated cells. Ultrastructural changes of the mitochondria affected the cristae, which became less electron dense and underwent fragmentation and even- 


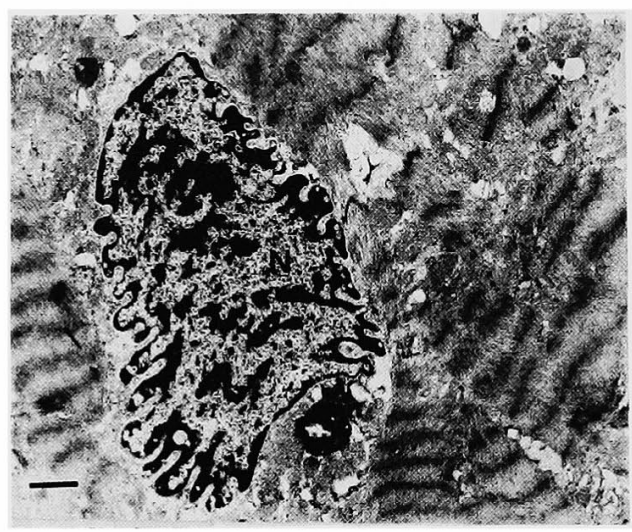

Fig. 5.

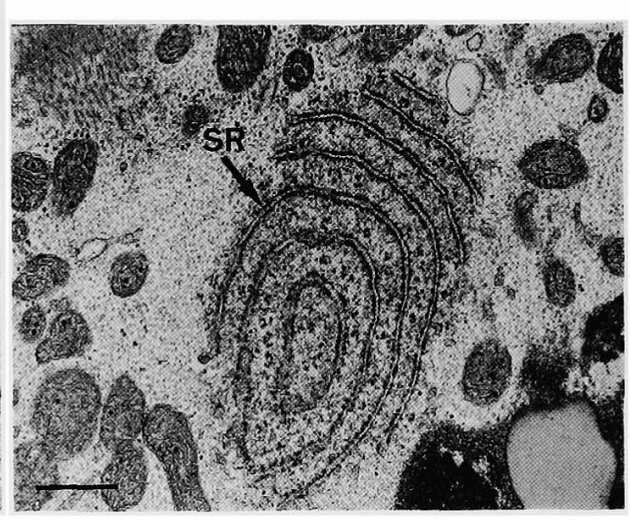

Fig. 6 .

Fig. 5. The bizarre-shaped nucleus (N) showing an increase in chromatin clumping at the nuclear margin. Horizontal bar $=1 \mu \mathrm{m}(\times 10,000)$.

Fig. 6. Proliferation of rough sarcoplasmic reticulum (SR). Horizontal bar $=0.5 \mu \mathrm{m}(\times 35,000)$.

tually disappeared. Sometimes the mitochondria became swollen and their matrix was more or less electron dense than normal. The normal electron dense mitochondrial granules also increased in number. Occasionally, glycogen granules were seen in the mitochondria. Vesicles, vacuoles, tubular structures, membranous and myelin bodies appeared in the mitochondria. More rarely inclusions occurred in mitochondria.

4. Nuclei and Golgi Apparatus. The nuclei frequently showed marked variation in shape and size and an increase in number of the nuclei was commonly seen (Fig. 5). The enlarged nuclei were often vesicular with the dense chromatin situated chiefly at the nuclear margin and they contained one or more prominent nucleoli. Frequently, the surfaces of the nuclei were indented. The nuclear membranes occasionally showed localized distension, focal disruption and loss. In some instances, the nuclei underwent degeneration and disintegration, even to the extent of necrosis. Closely associated with the nuclei was the Golgi apparatus which often presented more flattened tubules with terminal expansions.

5. Sarcoplasmic Reticulum and Transverse Tubular System. The ultrastructural alterations of the sarcoplasmic reticulum and transverse tubules were less frequently seen. The sarcoplasmic reticulum was distended. Proliferation of rough sarcoplasmic reticulum was also observed (Fig. 6). The transverse tubules were sometimes unusually long and dilated.

6. Other Elements of the Sarcoplasm. Lipid bodies and lipofuscin appeared in increased amounts and were found in large numbers in some cases (Fig. 7). In most cases the glycogen was increased. The glycogen 


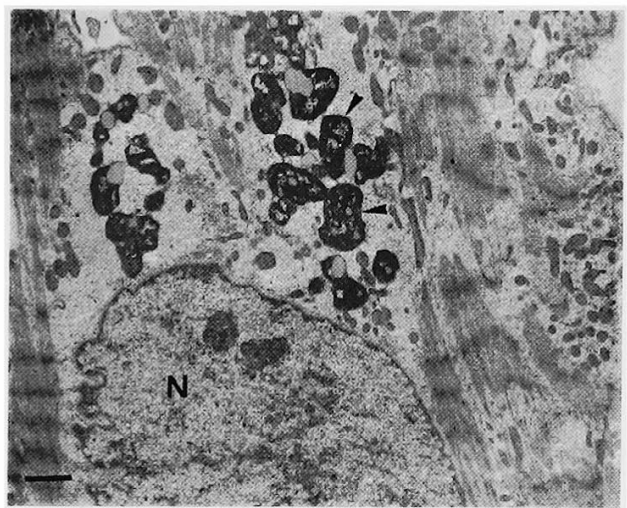

Fig. 7.

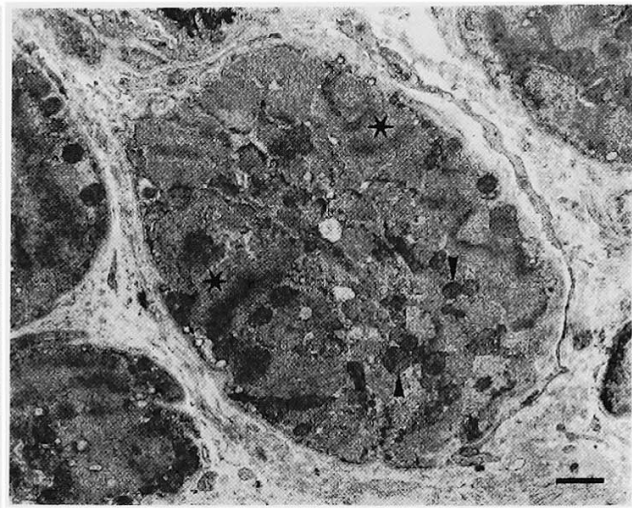

Fig. 8.

Fig. 7. An increased amount of lipofuscin (arrow heads) in the perinuclear region of the cell. $\mathrm{N}=$ nucleus. Horizontal bar $=1 \mu \mathrm{m}(\times 10,000)$.

Fig. 8. The myocardial cell in cross section showing normal subcellular structures. The myofibrils and mitochondria are indicated by asterisks and arrow heads respectively. Horizontal bar $=1 \mu \mathrm{m}(\times 10,000)$.

granules accumulated throughout the cells, especially between the myofibrils and under the sarcolemma. The glycogen particles occasionally appeared in the mitochondria, autophagic vacuoles and other membrane bound bodies. In cells in advanced stages of degeneration the glycogen granules disappeared along with the other organelles. Vacuoles of varying size were often observed and were very prominent in some cases. Myelin and membrane bodies were found in degenerating cells. They occurred under the sarcolemma, between myofibrils and within mitochondria or large membrane-bound vacuoles. The occurrence of various tubular structures was very rare.

Stage of ultrastructural pathological findings of myocardial cells: In order to correlate ultrastructural changes to the hemodynamic and echocardiographic data we arbitrarily examined 500 cells in cross section from each case. Based on the severity of the ultrastructural alterations of myofibrils and mitochondria which are two of the major subcellular structures, myocardial degenerative changes werc divided into a series of 5 stages as described in the following.

Stage I (Myocardial cells with normal or nearly normal ultrastructure). In this stage, the myocardial cells showed that the mitochondria were relatively evenly distributed throughout the cross section of myofilament masses without significant morphological changes (Fig. 8). Occasionally, some degenerative changes manifested by myofibrillar disarray and loss, particularly in the peripheral portion of sarcoplasm, were observed. 


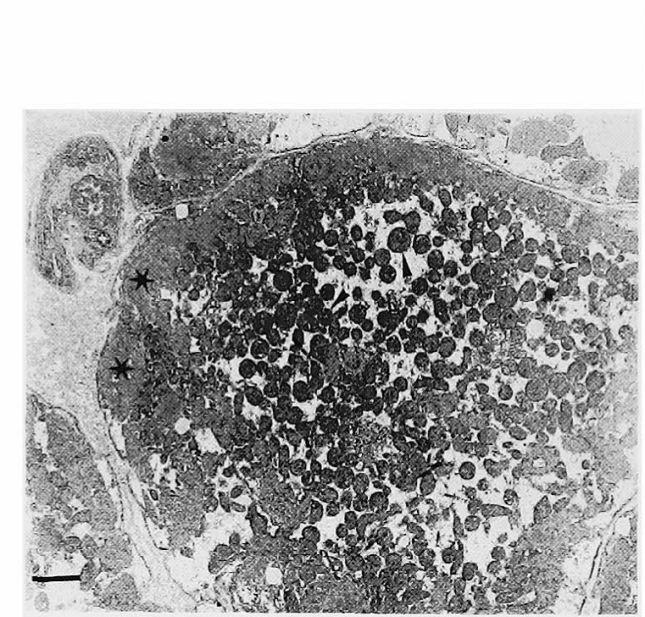

Fig. 9.

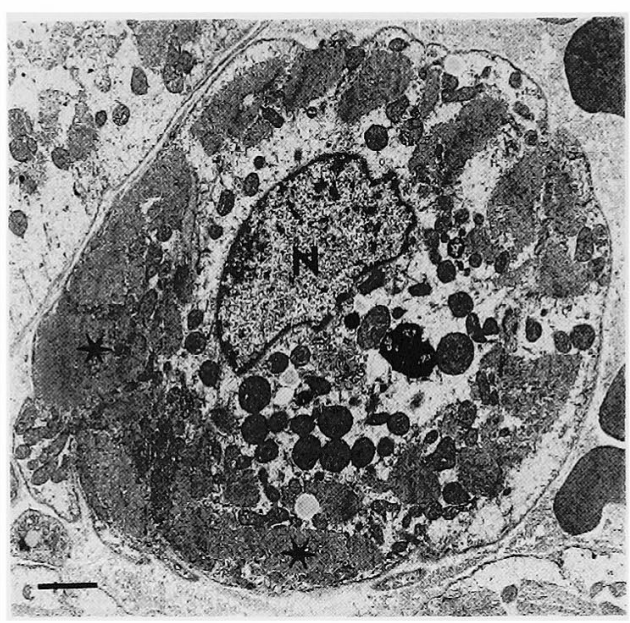

Fig. 10.

Fig. 9. The myocardial cell in cross section showing the disproportion of mitochondria (arrow heads) to myofibrils (asterisks) resulting from proliferation of the mitochondria. Horizontal bar $=2 \mu \mathrm{m}(\times 5,000)$.

Fig. 10. The myocardial cell in cross section showing myofibrillary degeneration with apparent loss of myofibrils (asterisks) in the perinuclear region of the cell. $\mathrm{N}=$ nucleus. Horizontal bar $=2 \mu \mathrm{m}(\times 6,000)$.

Stage II (The disproportion of mitochondria to myofibrils usually associated with mild degenerative changes of ultrastructure). The myocardial cells in this stage showed that the mitochondria occupied a greater percentage $(\geq 60 \%$ ) of the cross section of the cells usually associated with significant changes of mitochondrial ultrastructure (Fig. 9). In addition, some degree of myofibrillar disarray and loss were a consistent finding. This stage indicated early degenerative changes of myocardial cells.

Stage III (Severe myofibrillolysis always associated with apparent degenerative changes of the mitochondria). Areas of marked myofibrillary degeneration with apparent loss of myofibrils, particularly in the perinuclear and peripheral regions of the cells, were the primary pathological findings in this stage (Fig. 10). The normal myofibrillar masses were still present. A decrease in number of mitochondria always associated with variable degrees of degenerative changes of ultrastructure was frequently seen. This stage indicated advanced degenerative changes of myocardial cells.

Stage IV (The formation of sarcoplasmic masses without visualization of normal myofilament masses in the cells). In this stage severe degeneration of the myofibrils resulted in total loss of the myofibrils with the formation of sarcoplasmic masses which contained many glycogen particles, electrondense granular materials, mitochondria, occasional nuclei and remains of 


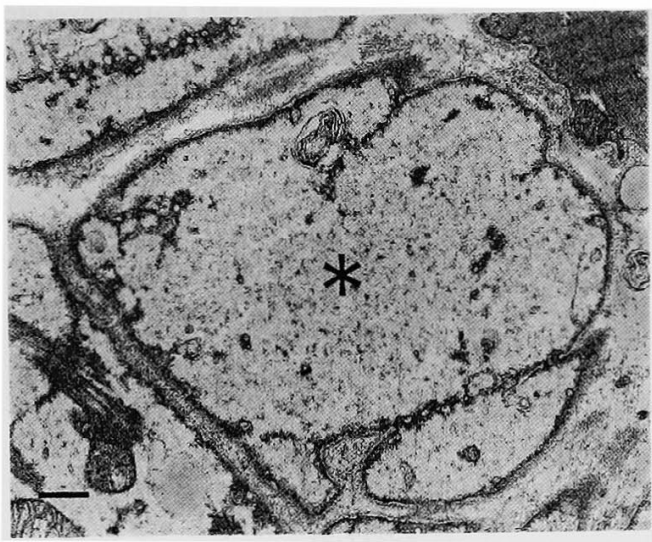

Fig. 11 .

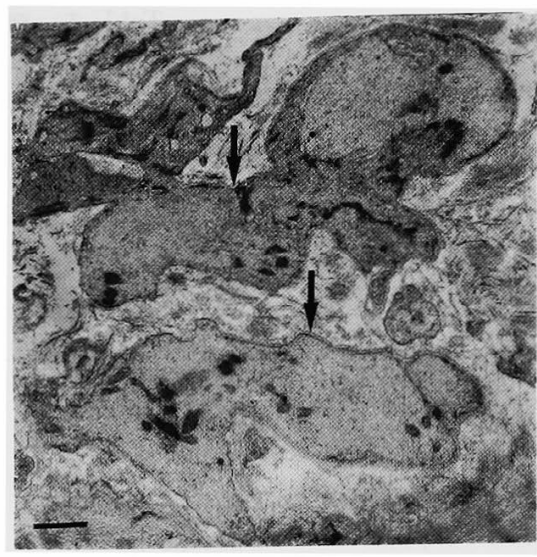

Fig. 12.

Fig. 11. The myocardial cell in cross section (asterisk) showing the formation of sarcoplasmic mass without visualization of myofilaments and mitochondria. Horizontal bar $=1 \mu \mathrm{m}(\times 10,000)$.

Fig. 12. The sclerotic changes of myocardial cells (arrows) with the sarcoplasmic masses and remains of degenerated myofibrils and mitochondria. Note that the myocardial cells become very small and rigid with irregular configuration. Horizontal bar $=1 \mu \mathrm{m}(\times 12,000)$.

degenerated myofibrils or myofilaments (Fig. 11). In general, normal configuration of the muscle cells was still preserved. This stage indicated far advanced degenerative changes of myocardial cells.

Stage V (The formation of sclerotic degenerative cells). The myocardial cells became very rigid and small in size as well as irregular in shape with the sarcoplasmic masses and remains of degenerated myofibrils and mitochondria (Fig. 12). This final stage indicated necrotic changes of myocardial cells.

From the viewpoint of ultrastructural pathology the cells in stage I are considered to be functionally normal or nearly normal while the cells in stages II and III have mild to moderate functional impairment. The cells in stage IV can no longer maintain functional integrity but they are probably still alive. However, the cells in stage $\mathrm{V}$ are morphologically not viable.

Correlation of ultrastructural pathological findings to hemodynamic and echocardiographic data: The number and percentage of the myocytes distributed in the 5 different ultrastructural stages in the 2 groups of patients with and without left ventricular dysfunction are summarized in Table I while the hemodynamic and echocardiographic data are shown in Table II. It is noteworthy that there was a significant difference in stage I between groups A and B. This finding indicates that the functional myocytes without signi- 
Table I. Statistical Analysis of Distribution of 500 Cardiomyocytes Patients with Normal (Group A) and Abnor-

\begin{tabular}{|c|c|c|c|c|}
\hline \multirow{3}{*}{ No of cases } & & & \multicolumn{2}{|r|}{ Stages } \\
\hline & \multicolumn{2}{|c|}{ I } & \multicolumn{2}{|c|}{ II } \\
\hline & A & B & A & B \\
\hline 1 & $247(49.4)$ & $165(33.0)$ & $90(18.0)$ & $40(8.0)$ \\
\hline 2 & $305(61.0)$ & $226(45.2)$ & $102(20.4)$ & $81(16.2)$ \\
\hline 3 & $355(71.0)$ & $208(41.6)$ & $78(15.6)$ & $133(26.6)$ \\
\hline 4 & $324(64.8)$ & $263(52.6)$ & $75(15.0)$ & $25(5.0)$ \\
\hline 5 & $438(87.6)$ & $205(41.0)$ & $27(5.4)$ & $183(36.6)$ \\
\hline 6 & $301(60.2)$ & $271(54.2)$ & $143(28.6)$ & $199(39.8)$ \\
\hline 7 & $349(69.8)$ & & $73(14.6)$ & \\
\hline 8 & $414(82.8)$ & & $25(5.0)$ & \\
\hline 9 & $425(85.0)$ & & $47 \quad(9.4)$ & \\
\hline Mean $\pm S D$ & $\begin{array}{c}350.89 \pm 60.73 \\
(71.18 \pm 13.8)\end{array}$ & $\begin{array}{c}223 \pm 44.6 \\
(36.12 \pm 7.22)\end{array}$ & $\begin{array}{c}73.33 \pm 35.2 \\
(14.67 \pm 7.04)\end{array}$ & $\begin{array}{l}110.17 \pm 22.03 \\
(66.76 \pm 13.35)\end{array}$ \\
\hline$P$ value & $(0.001$ & $\begin{array}{l}0.001 \\
0.01)\end{array}$ & $\begin{array}{r}0.2 \\
(0.2\end{array}$ & $\begin{array}{l}<0.3 \\
<0.3)\end{array}$ \\
\hline
\end{tabular}

The number in the table indicates the number of cardiomyocytes in different stages.

The number in brackets indicates the percentage of cardiomyocytes in different stages.

Table II. Statistical Analysis of Echocardiographic and Hemodynamic Data in

\begin{tabular}{|c|c|c|c|c|}
\hline & & & \multicolumn{2}{|c|}{ Echocardiographic data } \\
\hline & \multicolumn{2}{|c|}{ LAD (mm) } & \multicolumn{2}{|c|}{ LVD (ESD/EDD) (mm) } \\
\hline & $A$ & B & A & B \\
\hline Mean $\pm S D$ & $47.67 \pm 8.08$ & $52.2 \pm 7.19$ & $\begin{aligned} 27.44 & \pm 5.42 \\
& \sim 40.33 \pm 5.19\end{aligned}$ & $\begin{aligned} 30 & \pm 6.84 \\
& \sim 40.4 \pm 9.09\end{aligned}$ \\
\hline$P$ value & 0.3 & 0.4 & $\begin{array}{r}0.4< \\
\sim\end{array}$ & \\
\hline
\end{tabular}

Abbreviations : $L A D=$ left atrial dimension ; $L V D=$ left ventricular dimension ; $E S D=$ end-systolic dimension; $\mathrm{EDD}=$ end-diastolic dimension; $\mathrm{MVA}=$ mitral valve area; $\mathrm{PAWP}=$ pulmonary

ficant ultrastructural changes were much more decreased in number in patients with abnormal left ventricular function. In addition, an advanced stage of myocyte injury was also observed primarily in patients with impaired left ventricular function. There were no significant differences in hemodynamic and echocardiographic data between groups $\mathrm{A}$ and $\mathrm{B}$, indicating that left ventricular dysfunction in patients with mitral stenosis is not related to the severity of mitral stenosis. 
in Five Different Stages of Ultrastructural Pathological Findings in mal (Group B) Left Ventricular Function

\begin{tabular}{|c|c|c|c|c|c|}
\hline \multicolumn{2}{|c|}{ III } & \multicolumn{2}{|r|}{ IV } & \multicolumn{2}{|r|}{$\mathrm{V}$} \\
\hline A & B & A & B & $\mathrm{A}$ & B \\
\hline $\begin{array}{r}152(30.4) \\
93(18.6) \\
67(13.4) \\
101(20.2) \\
35(7.0) \\
56(11.2) \\
78(15.6) \\
61(12.2) \\
28(5.6)\end{array}$ & $\begin{array}{r}186(37.2) \\
136(27.2) \\
126(25.2) \\
181(36.2) \\
96(19.2) \\
30(6.0)\end{array}$ & $11(2.2)$ & 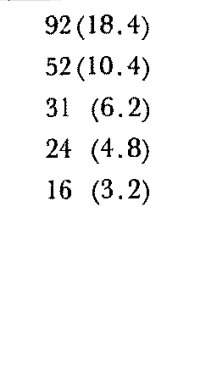 & & $\begin{array}{r}17(3.4) \\
5(1.0) \\
2(0.4) \\
7(1.4)\end{array}$ \\
\hline $\begin{array}{r}74.56 \pm 35.54 \\
(14.9 \pm 7.11) \\
0.05 \\
(0.05\end{array}$ & $\begin{array}{l}125.83 \pm 25.17 \\
(52.98 \pm 10.6) \\
<0.1 \\
<0.1)\end{array}$ & & $\begin{array}{c}43 \pm 6.6 \\
(27.26 \pm 2.55)\end{array}$ & & $\begin{array}{c}7.75 \pm 1.55 \\
(5.63 \pm 1.13)\end{array}$ \\
\hline
\end{tabular}

Mean $\pm S D=$ mean \pm standard deviation.

Patients with Normal (Group A) and Abnormal (Group B) Left Ventricular Function

\begin{tabular}{|c|c|c|c|c|c|}
\hline & & \multicolumn{4}{|c|}{ Hemodynamic data } \\
\hline \multicolumn{2}{|c|}{$\operatorname{MVA}\left(\mathrm{cm}^{2}\right)$} & \multicolumn{2}{|c|}{ PAWP (mmHg) } & \multicolumn{2}{|c|}{ LVP (PSP/EDP) (mmHg) } \\
\hline $\mathrm{A}$ & $\mathrm{B}$ & A & $\mathrm{B}$ & A & B \\
\hline \multicolumn{2}{|c|}{$0.2<\mathrm{p}<0.3$} & \multicolumn{2}{|c|}{$p>0.5$} & $\begin{array}{r}108.11 \pm 13.28 \\
\sim 9.89 \pm 2.96 \\
\mathrm{p}>0.5 \\
\sim \mathrm{p}>\end{array}$ & $\begin{aligned} 104 & \pm 8.81 \\
& \sim 9 \pm 2.71\end{aligned}$ \\
\hline
\end{tabular}

artery wedge pressure; $L V P=$ left ventricular pressure; $P S P=$ peak systolic pressure ; $E D P=$ enddiastolic pressure.

\section{Discussion}

A number of studies have provided unequivocal evidence for the high incidence of impairment of left ventricular function in patients with isolated rheumatic mitral stenosis. ${ }^{6), 12)-14)}$ However, the pathophysiological mechanisms responsible for left ventricular dysfunction in these patients still remain poorly understood. It has been suggested that abnormal left ventricular function in patients with mitral stenosis results from the hemodynamic 
derangement, the underlying coronary artery disease and the presence of a myocardial factor. $\left.\left.{ }^{1)-3(, 6)-9}, 12\right), 15\right\}, 16$ ) In the present report we did not find any correlation between the abnormalities in left ventricular function as assessed by echocardiography and angiocardiography and the severity of mitral stenosis reflected in the hemodynamic and echocardiographic parameters. These findings indicate that hemodynamic factors do not contribute greatly to the development of left ventricular dysfunction. In addition, the normal coronary angiography without electrocardiographic evidence of myocardial infarction observed in all patients excluded the possibility that the underlying coronary artery disease played a significant role in the pathogenesis of the impaired left ventricular function. Thus, the presence of a myocardial factor should be considered the primary pathogenetic mechanism responsible for abnormal left ventricular function in patients with mitral stenosis in the present study.

Unfortunately, the available data supporting a myocardial factor as the primary cause of left ventricular dysfunction are far from complete. Although previous histopathological studies have shown that the failure of improvement after mitral commissurotomy was related to the extent of myocardial involvement by the rheumatic process ${ }^{9}{ }^{9}$ there is still no systematic report dealing with the ultrastructural pathological changes of myocardial cells in patients with mitral stenosis. Regardless of whether normal or impaired left ventricular function was present in patients with mitral stenosis, we have consistently demonstrated varying degrees of pathological alterations of myocardial ultrastructure in all specimens examined. The patients with mitral stenosis and left ventricular dysfunction showed a greater amount of myocyte injury and loss. Furthermore, a disproportion of the mitochondriato-myofibril ratio and severe myofibrillolysis with the formation of sarcoplasmic masses were observed primarily in patients with impaired left ventricular function. These findings, taken together, suggest that generalized abnormalities of myocardial ultrastructure persist following the rheumatic process in patients with rheumatic mitral stenosis. The damage of myocardial cells resulting from loss of myofibrils due to either mitochondrial proliferation or myofibrillar degeneration may be the main cause of contractile dysfunction of the left ventricular myocardium.

It is common to all tissues that they can rapidly adapt their structural design whenever sustained changes of activity and/or load occur, though within limits and in different ways. In a disease like mitral stenosis the hemodynamic burden of the mechanical obstruction is on the pulmonary circulation and right heart chambers. The reverse types of structural adaptation in left ventricular myocardium may occur in response to sustained de- 
creases of hemodynamic burden in the left ventricle. However, ultrastructural pathological findings of the left ventricular myocardium observed in patients with mitral stenosis did not correlate with the hemodynamic data of the left heart. Thus, we suggest that pathological alterations of myocardial ultrastructure observed in chronic rheumatic mitral stenosis were related to the extent of myocardial involvement by the rheumatic process rather than being a structural adaptation in response to the hemodynamic derangement.

In summary, we have consistently demonstrated varying degrees of ultrastructural pathological alterations of left ventricular myocardium in all patients with isolated rheumatic mitral stenosis. The ultrastructural pathological findings did not correlate with the severity of mitral stenosis reflected in the echocardiographic and hemodynamic data. However, the patients with abnormal left ventricular function always exhibited a more extensive loss of myofibrils resulting from either disproportion of the mitochondria-tomyofibril ratio or myofibrillar degeneration. From these results, the present investigation provides the morphological data at the ultrastructural level to support the widely held concept of a myocardial factor i.e., the extent of myocardial involvement by the rheumatic process as the basic pathogenetic mechanism responsible for left ventricular dysfunction in patients with isolated rheumatic mitral stenosis.

\section{REFERENGES}

1. Heller SJ, Carleton RA: Abnormal left ventricular contraction in patients with mitral stenosis. Girculation 42: 1099, 1970

2. Curry GC, Eliott LP, Ramsey HW: Quantitative left ventricular angiographic findings in mitral stenosis. Am J Cardiol 29: 621, 1972

3. Holtzer JA, Karliner JS, O'Rourke RA, Peterson KL: Quantitative angiographic analysis of the left ventricle in patients witl isolated rheumatic mitral stenosis. Br Heart J 35:497, 1973

4. Horwitz LD, Mullins CB, Payne RM, Curry GC: Left ventricular function in mitral stenosis. Chest 64: 609, 1973

5. Dubiel JP, Dubiel JS: Quantitative angiocardiography evaluation of left ventricular function in mitral stenosis. Pol Med Sci Hist Bull 15: 183, 1975

6. Ibrahim MM: Left ventricular function in rheumatic mitral stenosis: Clinical echocardiographic study. Br Heart J 42: 514, 1979

7. Silverstein DM, Hansen DM, Hansen DP, Ojiambo HP, Griswold HR: Left ventricular function in severe pure mitral stenosis as seen at the Kenyatta National Hospital. Am Heart. J $99: 727,1980$

8. Rao PS, Mukhopadhyag S, Tandon R, Shrivastava S: Left ventricular function in isolated rheumatic mitral stenosis in children. Pediatr Cardiol 6: 293, 1986

9. Selim NM: Studies on rheumatic mitral valve disease with special emphasis on the myocardial factor. M.D. Thesis, Assiut University, Egypt, 1976

10. London E, Popp RL: Reliability and reproducibility of two-dimensional echocardiographic measurement of stenotic mitral valve orifice area. Am J Cardiol 43:560, 1979

11. Langer GA: The structure and function of the myocardial cell surface. Am J Physiol 235: 
$\mathrm{H} 461,1978$

12. Harvey RM, Ferrer MI, Samet P, Bader RA, Bader ME, Cournard A, Richards DW: Mechanical and myocardial factors in rheumatic heart disease with mitral stenosis. Circulation 11: 531,1955

13. Kennedy JW, Yarnall SR, Murray JA, Figley MM: Quantitative angiocardiography. IV. Relationships of left atrial and ventricular pressure and volume in mitral valve disease. Circulation 41: 817,: 1970

14. McDonald IJ: Echocardiographic assessment of left ventricular function in mitral disease. Circulation 53: 865, 1976

15. Befeler B, Kamen AR, MacLeod CA: Coronary artery disease and left ventricular function in mitral stenosis. Chest 57: 435, 1970

16. Feigenbaum H, Campbell RW, Wunsch CM, Steinmetz EF: Evaluation of the left ventricle in patients with mitral stenosis. Girculation 34: 462, 1966 